\title{
La transposition de quaestor en grec
}

Il existe plusieurs manières d'aborder l'étude de la transposition en grec du vocabulaire des institutions politiques et de la vie publique romaines. On peut en distinguer trois, qui entraînent l'adoption de méthodes radicalement différentes.

La première consiste à examiner un seul terme et à en faire une étude diachronique complète. Elle peut prendre la forme d'une monographie sur un concept, un champ sémantique, une magistrature, etc. Du point de vue institutionnel, l'exemple le plus achevé est le travail que M. Holleaux a consacré au titre primitif du consul en grec, $\sigma \tau \rho \alpha \tau \eta \gamma o ̀ s$ ט̈ $\pi \alpha \tau \circ \varsigma^{1}$. L'originalité de cette étude, qui est un modèle du genre, est d'avoir pleinement exploité avec bonheur les témoignages littéraires (surtout Polybe) et les données épigraphiques d'un dossier complexe. Il faut regretter que cette voie de recherche, riche d'enseignements, fasse encore aujourd'hui figure d'exception.

La seconde approche consiste à limiter la recherche à un seul auteur. On dispose ainsi, pour certains d'entre eux, d'études particulières qui privilégient tantôt l'aspect linguistique (l'influence du latin sur le grec) $)^{2}$, tantôt l'aspect institutionnel (par exemple, la transposition du nom des magistratures) ${ }^{3}$. Ces ouvrages, peu nombreux au demeurant, laissent souvent à désirer, car les instruments de travail indispensables, en particulier les index et les concordances d'auteurs, ont longtemps fait défaut pour aborder l'œuvre des historiens grecs de Rome ${ }^{4}$. Toute étude

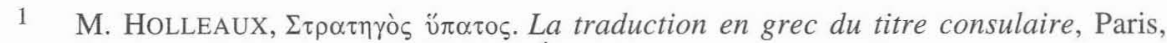

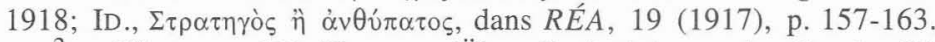

2 Cf., p. ex., J.B. Ullrich, Über die Latinismen des Cassius Dio, Heidelberg, 1912; J. Hering, Lateinisches bei Appian, Leipzig, 1935. Pour Polybe, cf. infra n. 6.

3 V. NORDSTRÖM, De institutorum Romanorum vocabulis Dionysii Halicarnassensis quaestiones, Helsinki, 1890; G. VRIND, De Cassii Dionis vocabulis quae ad ius publicum pertinent, La Haye, 1923; M.-L. FREYBURGER-GAlland, Aspects du vocabulaire politique et institutionnel de Dion Cassius, Paris, 1997; D. RoQues, Le vocabulaire politique d'Hérodien, dans Ktèma, 15 (1990), p. 35-71; Ét. FAMERIE, Le latin et le grec d'Appien. Contribution à l'étude du lexique d'un historien grec de Rome, Genève, 1998.

4 Cf. A. Mauersberger et al., Polybius-Lexicon, 5 vol. parus, Berlin, 1956-1998 $\left(\alpha-\pi \circ \varepsilon \varepsilon_{\omega}\right)$, I. McDougall, Lexicon in Diodorum Siculum, 2 vol., Hildesheim, 1973 (mais les livres fragmentaires XXI-XL, consacrés essentiellement à l'histoire romaine, ne sont pas dépouillés!); K.H. Rengstorf et al., A Complete Concordance to Flavius Josephus, 4 vol., Leyde, 1973-1983; Ét. FAmerie, Concordantia in Appianum, 3 vol., Hildesheim, 1993; W. NawiJn, Cassii Dionis Cocceiani Historiarum Romanarum index graecitatis, Berlin, 1931; A.C. BANDY, Ioannes Lydus, On Powers or The Magistracies of the Roman 
entreprise dans ces conditions ne pouvait se fonder que sur des dépouillements sélectifs. En matière de lexicologie, si l'exhaustivité n'offre pas la garantie d'une démarche pertinente, elle conditionne beaucoup les ambitions et les limites de la recherche 5 .

Trop souvent aussi, de telles études négligent l'aspect historique de la recherche. À cet égard, les travaux de M. Dubuisson sur Polybe ont montré tout l'intérêt qu'il y avait à envisager conjointement les deux points de vue ${ }^{6}$. Il a jeté, le premier, les fondements théoriques d'une étude du bilinguisme gréco-romain pour les appliquer à un cas particulier, celui de Polybe. Il a ainsi pu dégager les implications historiques du bilinguisme du plus ancien historien grec de Rome que nous puissions lire.

La troisième approche consiste à envisager le vocabulaire institutionnel dans son ensemble, en se fondant sur le corpus le plus vaste qui soit (sources littéraires, inscriptions et papyrus). En principe, c'est la démarche la plus ambitieuse, qui devrait s'effectuer en dernier lieu. Paradoxalement, c'est par là que la recherche a pour ainsi dire commencé, en tentant de fournir d'emblée des synthèses, sans même disposer d'études particulières fiables. À titre d'exemple, $M$. Mentz ${ }^{7}$ parvenait déjà à la conclusion que l'étude du lexique «romain» des historiens grecs de Rome permettrait de distinguer, parmi eux, les novateurs (Polybe, Denys, Dion Cassius) et les conservateurs (Diodore, Josèphe, Plutarque, Appien).

En l'état actuel de nos connaissances, tout indique qu'une étude aussi ambitieuse est encore prématurée. Elle pourra seulement être entreprise avec quelque chance de succès quand on disposera d'études particulières qui se fondent sur une analyse raisonnée du phénomène de la transposition. Il vaut la peine d'examiner brièvement l'économie des ouvrages relatifs à cette question, car les meilleurs ont acquis au fil du temps le statut de dictionnaires de référence pour l'étude du lexique des historiens grecs de Rome.

State, Philadelphie, 1983. En revanche, pour Denys d'Halicarnasse, Nicolas de Damas, Strabon, Plutarque, Hérodien et Zosime, on ne dispose que de modestes index dans les éditions. À l'heure actuelle, seul le Thesaurus linguae Graecae (CD-ROM D) permet de pallier cette lacune.

5 On en trouvera une illustration dans l'édition de l'Histoire nouvelle de Zosime par Fr. PAschoud. Dans un premier temps, l'éditeur (t. I, CUF, Paris, 1971, p. LXXII-LXXV), regrettant l'absence d'index verborum, se borna à livrer quelques réflexions désabusées sur l'incohérence du lexique des «antiquités» de Zosime. Au cours du travail d'édition, il entreprit de réaliser à son usage personnel un tel lexique (publié t. III, 2, 1989, p. 201212), dont il avoue qu'il aurait tiré grand profit s'il en avait disposé dès le début.

6 M. Dubuisson, Le latin de Polybe. Les implications historiques d'un cas de bilinguisme, Paris, 1985.

7 Cf. infra n. 8. 


\section{Les lexiques grecs des institutions romaines}

\section{M. Mentz}

La thèse de M. Mentz (De magistratuum Romanorum Graecis appellationibus) affiche comme ambition l'étude du nom des magistrats romains dans les sources grecques ${ }^{8}$. Les onze chapitres consacrés aux différentes magistratures ne traitent le sujet annoncé que sommairement et comportent plusieurs défauts majeurs. M. Mentz avait pour objectif de dresser un tableau général du vocabulaire institutionnel à partir d'une matière qu'il ne pouvait guère dominer (dix auteurs), en l'absence d'outils adéquats ${ }^{9}$. Son exposé repose sur un choix de témoignages restreint, qui débouche, en définitive, sur une brève description de quelques termes chez les auteurs. De plus, M. Mentz n'a guère exploité les documents épigraphiques réunis peu avant par P. Viereck ${ }^{10}$. Il se contente d'en invoquer quelques-uns pour confirmer l'analyse des sources littéraires. Là encore, la simple juxtaposition de références ne rend compte en aucune manière d'un système organisé.

\section{D. Magie}

Dix ans plus tard, l'étude du lexique grec des institutions romaines connut une étape décisive grâce à la publication du lexique de D. Magie (De Romanorum iuris publici sacrique vocabulis sollemnibus in Graecum sermonem conversis $)^{11}$. L'ouvrage, différent de celui de M. Mentz et beaucoup plus complet, se présente comme un véritable dictionnaire latin-grec du vocabulaire politique, militaire et religieux du monde romain (République et Empire).

D. Magie jeta aussi les bases théoriques d'une étude de la transposition en grec des réalités romaines. Au terme de son analyse, il dressait une typologie selon laquelle la transposition en grec peut s'effectuer de trois manières, par équivalence (comparatio), transcription (transcriptio) ou traduction (interpretatio). Pour illustrer son analyse, il fournit un exemple devenu classique : en grec, quaestor peut se dire $\tau \alpha \mu i \alpha_{\varsigma}$ par

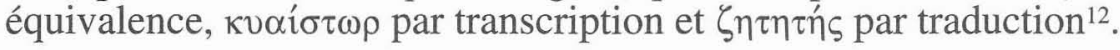

8 Iéna, 1894, 51 p. Avant cette date, seules les sources littéraires avaient été exploitées: C.Fr. WEBER, Dissertatio de Latine scriptis quae Graeci ueteres in suam linguam transtulerunt, 4 vol., Kassel, 1835-1852; A. WANNOWSKI, Antiquitates Romanae e Graecis fontibus explicatae, Königsberg, 1846.

9 Polybe, Denys, Diodore, Strabon, Josèphe, Plutarque, Appien, Dion Cassius, Hérodien et Jean le Lydien, pour lesquels il ne disposait que de lexiques sommaires.

10 P. VIERECK, Sermo Graecus quo senatus populusque Romanus magistratusque populi Romani usque ad Tiberii aetatem in scriptis publicis usi sunt examinatur, Göttingen, 1888.

11 Leipzig, 1905, 183 p.

12 D. Magie, $R V$, p. 2; pour l'exposé détaillé sur les trois méthodes, cf. $R V$, p. 3-41. 
Dans son introduction, D. Magie souligne à juste titre que les trois procédés ne sont pas employés de manière indifférente. Dans les textes littéraires, le mode normal de transposition est l'équivalence, alors que le recours à la transcription y est exceptionnel, sauf pour quelques termes (p. ex. $\delta i \kappa \tau \alpha ́ \tau \omega \rho)^{13}$. En revanche, si l'équivalence reste courante dans les inscriptions et dans les papyrus ${ }^{14}$, la transcription y est aussi fréquente. D. Magie concluait à l'existence d'un mode de transposition privilégié dépendant de la nature des témoignages (littéraires ou documentaires).

Cette analyse pertinente ne se retrouve cependant pas dans le corps du lexique. À chaque lemme, on voit se côtoyer des références à des auteurs que sept siècles séparent (de Polybe à Jean le Lydien), à des inscriptions d'époque républicaine ou impériale de nature diverse (traductions de textes officiels, inscriptions honorifiques, dédicaces, épitaphes) et au Corpus glossarum Latinarum. Aussi n'est-il pas rare de trouver dans son lexique dix expressions grecques transposant un même terme latin.

Le défaut de l'ouvrage est donc double. D'une part, l'absence de traitement d'une telle masse de témoignages ne permet pas de distinguer les particularités d'emploi des différents termes ou expressions bien attestés ${ }^{15}$. D'autre part, l'ouvrage étant un lexique latin-grec, la matière y est organisée autour de notions et de réalités romaines. Une telle présentation, qui privilégie le latin, empêche de vérifier la cohérence de la terminologie grecque.

La richesse de ce lexique lui conféra d'emblée le statut d'ouvrage de référence et en fit presque un répertoire officiel de la terminologie grecque des institutions romaines ${ }^{16}$. Malgré ses qualités, le lexique

13 Le mot était d'autant plus facile à adopter en transcription que les deux langues possèdent le même suffixe de nom d'agent $(-\tau \omega \rho,-$-tor $)$. Une différence doit cependant être soulignée. En latin, le suffixe était caractérisé, à l'origine, par l'alternance entre voyelle longue au nominatif et voyelle brève aux autres cas; à date historique, la voyelle longue devant $r$ et en syllabe finale s'est abrégée (cf. A. ERnout, Morph. hist. lat., Paris, 195332, $\S 55)$, ce qui aboutit à un modèle de déclinaison différent du grec $(-\tau \omega \rho,-\tau \rho \rho \circ \varsigma$, mais -tŏr -tōris). Cf. infra, n. 36.

14 En 1905, D. Magie ne pouvait guère exploiter le témoignage des papyrus. Leur analogie avec les inscriptions ne sera mise en évidence que plus tard, en particulier par H.J. Mason (cf. infra p. 215-217).

15 Seul figure à côté de chaque expression un chiffre romain symbolisant le mode de transposition : équivalence (I), traduction (II), transcription (III). Mais, dans bien des cas, on trouve aussi des notations comme (I, II), (I, III), (II, III), (II, III ?), qui sont difficiles à interpréter.

16 Le système est repris sans discussion par F. VISCIDI, I prestiti latini nel greco antico e bizantino, Padoue, 1944, p. 11. Aujourd'hui encore, on s'y réfère souvent comme à un axiome; cf. R.K. SHERK, Roman Documents from the Greek East, Baltimore, 1969, p. 15-16: «David Magie has long ago demonstrated the principles whereby the titles of Roman magistrates were expressed in Greek. (...) All three of these methods are used in the senatus consulta.» Plus récemment, cf. M.-L. Freyburger-Galland, o.l. [cf. n. 3], 
repose sur une économie différente de celle d'un dictionnaire bilingue moderne et sa consultation exige une grande prudence, dont on se dispense trop souvent. Pour mieux assurer la démarche, il est indispensable de reprendre 1'examen de la transposition en grec du lat. quaestor (l'exemple canonique de D. Magie). Comme on le verra plus loin, il n'est pas sans incidence sur le plan méthodologique ${ }^{17}$.

\section{H.J. Mason}

En 1974, H.J. Mason publia un ouvrage qui était appelé à remplacer en partie le lexique de D. Magie : Greek Terms for Roman Institutions. A Lexicon and Analysis ${ }^{18}$.

Tout d'abord, H.J. Mason a renoncé au classement de son prédécesseur, auquel il reproche de ne pas tenir compte des particularités d'emploi des trois modes de transposition. Il a aussi modifié l'économie du lexique sur un point plus important, en prenant pour lemmes les termes grecs. La première place accordée au grec permet de mieux saisir la variété des emplois d'un même terme. Ce constat l'a incité à ne pas seulement fournir un outil qui aurait pu être, somme toute, l'image inversée de celui de D. Magie, mais à y joindre une série de chapitres consacrés à l'analyse de mots ambigus.

L'auteur souligne le danger de prendre pour équivalents l'ensemble des témoignages littéraires, épigraphiques et papyrologiques et d'en tirer des conclusions sans tenir compte de leur nature. Plusieurs pages de sa préface esquissent une réflexion consacrée à ce qu'on pourrait appeler une «approche différenciée» des témoignages, en examinant les principaux critères à observer pour présenter les données. L'influence du latin peut varier en fonction de divers aspects.

\section{- nature et fonction des documents}

Il faut distinguer les documents à caractère officiel (lois, traités, sénatus-consultes, lettres de magistrats, etc.) et privé (cursus sénatoriaux et équestres, dédicaces, épitaphes, etc.). Cette précaution est indispensable pour déterminer jusqu'à quel point des textes de nature et de portée aussi différentes peuvent faire l'objet d'une même exploitation lexicologique.

p. 27 : «On s'aperçoit que le traducteur a, selon ses motivations, ses compétences, ses goûts stylistiques, trois solutions : il pourra recourir, selon la distinction traditionnelle, à une transcription littérale, une véritable traduction ou une équivalence.» La phrase résume bien la manière dont la théorie de Magie est encore reçue : Dion Cassius (appelé maladroitement «traducteur») est présenté comme ayant presque toujours le choix entre trois solutions pour transposer un même terme latin.

17 Cf. infra, p. 224-225.

18 Toronto, 1974, 207 p. 


\section{- domaine envisagé}

Par exemple, les transcriptions sont beaucoup plus fréquentes dans le domaine de la vie religieuse (dont le vocabulaire est peut-être le moins transférable de tous) ou militaire (où le besoin de précision répond au souci d'efficacité) que politique.

- diversité des témoignages littéraires et contraintes du genre historiographique

On ne contestera pas que Polybe se situe, à tous égards, aux antipodes de Jean le Lydien, tant au point de vue de la chronologie que de la démarche. Encore faut-il tenter de mesurer l'écart, en tenant compte du statut des auteurs, de leur entourage, de leur époque, etc.

Un autre phénomène particulier rend l'étude délicate, celui des contraintes littéraires inhérentes au genre historiographique. Comme le souligne H.J. Mason, la transposition dans une langue de réalités qui lui sont étrangères est, à coup sûr, le domaine auquel un écrivain peut offrir le plus de «résistance» ${ }^{19}$. L'écart par rapport à une norme (ou une tendance) définie à partir des documents officiels ne traduit en rien le manque de précision d'un système linguistique. Ainsi, les inscriptions montrent que consul a d'abord été rendu en grec par l'expression

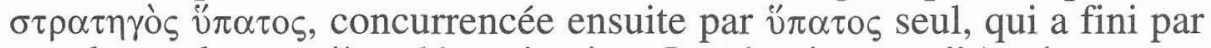
supplanter la première dénomination. Le témoignage d'Appien est en accord avec cette analyse, mais l'auteur ne s'en tient pas pour autant à un

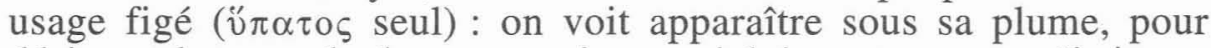
désigner le consul, des expressions qui lui sont propres ('̇ंท́㇒ı

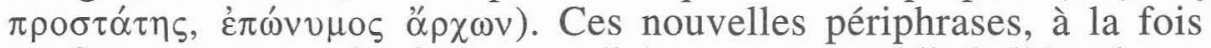
conformes au grec classique et explicites pour un public hellénophone, doivent s'analyser autrement que l'équivalent $\ddot{\pi} \pi \alpha \tau$ s, la traduction

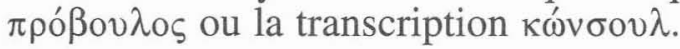

En conclusion, la méthode exposée par H.J. Mason dans sa préface est excellente. L'ouvrage marque un progrès certain, mais sur des points limités : exploitation plus systématique des documents épigraphiques et surtout papyrologiques, adoption d'un classement fondé sur les mots grecs, étude détaillée des termes présentant un grand degré de polysémie.

Malheureusement, le plan esquissé par Mason dans sa préface n'a guère servi de guide pour la rédaction des articles du lexique, qui reste, en définitive, proche du travail de D. $\mathrm{Magie}^{20}$. De plus, si l'auteur a raison de ne plus utiliser les gloses du $C G L$, on ne comprend pas qu'un

19 Cf. H.J. Mason, The Roman Government in Greek Sources. The Effect of Literary Theory on the Translation of Official Titles, dans Phoenix, 24 (1970), p. 150-159.

20 Par exemple, l'auteur donne à certains termes grecs une signification qui doit beaucoup au système de D. Magie, auquel il se réfère plus d'une fois. 
historien aussi important que Polybe soit volontairement tenu à l'écart du corpus (qui commence donc avec Diodore).

\section{E. García Domingo}

En 1979, E. García Domingo publia une étude philologique approfondie des documents officiels romains conservés en traduction grecque : Latinismos en la koiné (en los documentos epigráficos desde el 212 a. J.C. hasta el 14 d. J.C. $)^{21}$. L'auteur vise à donner une description et une analyse aussi complète que possible de tous les faits de langue observés. Mais il se fonde sur un corpus pour le moins curieux, sans fournir la moindre explication. Son étude porte en effet sur les sénatusconsultes édités par R.K. Sherk (32 documents) ${ }^{22}$, les lois, les traités et la version grecque des Res gestae; en revanche, les lettres de magistrats de la même période (46 documents) ont été écartées de manière tout à fait injustifiée ${ }^{23}$.

L'ouvrage comporte deux sections. La première fournit un exposé de tous les phénomènes d'ordre phonétique, morphologique et syntaxique; la seconde contient un lexique grec-latin du vocabulaire commun et divers index complémentaires (latin-grec, noms de personnes, de dieux, de lieux).

Certains ont reproché à E. García Domingo de fournir, sur chaque point, une simple liste d'exemples sans guère les exploiter ${ }^{24}$. S'il faut aussi regretter que l'auteur n'ait pas confronté son analyse avec le corpus étudié pour tenter d'en améliorer le texte, on possède néanmoins grâce à lui un ouvrage de référence, aux ambitions limitées, qui complète l'étude toujours utile de P. Viereck ${ }^{25}$.

Les ouvrages de D. Magie et de H.J. Mason doivent être utilisés avec une grande prudence, car, s'ils se présentent comme des lexiques bilingues, aucun n'est conçu comme un dictionnaire moderne de traduction. Au mieux, ils fournissent le matériau brut que l'utilisateur

21 Burgos, 1979,845 p.

22 Je ne m'explique pas la présence, dans l'ouvrage de R.K. SHERK, RDGE, du dernier document (32), qui date de 23 apr. J.-C. (sénatus-consulte relatif à l'Héraion de Samos et à l'Asclépieion de Cos).

23 Le recueil que j'ai constitué en prévision d'une refonte des $R D G E$ montre combien les progrès de l'épigraphie sont rapides et substantiels. L'ouvrage de Domingo, malgré sa date relativement récente (1979), est dépassé sur bien des points, à cause de la publication de nouvelles éditions critiques ou de la découverte de nouveaux documents (en particulier le riche dossier d'Aphrodisias : cf. J. REYNOLDS, Aphrodisias and Rome, Londres, 1982, $\mathrm{n}^{\circ} 1$ 13). Pour les lois épigraphiques d'époque républicaine conservées en traduction grecque, cf. désormais M.H. CRAWFORD et al., Roman Statutes, t. I, Londres, 1996.

24 Cf. les comptes rendus d'E. CAMPAnILE, dans Athenaeum, 59 (1981), p. 568-570;

U. Rapallo, dans Maia, 34 (1982), p. 91-93.

25 P. VIERECK, op. cit. (n. 10). 
doit lui-même organiser en article de dictionnaire. À titre d'illustration, un nouvel examen de la transposition de quaestor est éloquent ${ }^{26}$.

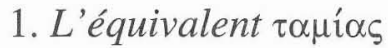

La transposition la plus courante de quaestor est l'équivalent $\tau \alpha \mu i \alpha \varsigma$, qui désigne, dans de nombreuses cités grecques, un magistrat dont les fonctions de trésorier-payeur et d'intendant évoquent en partie celles du questeur romain ${ }^{27}$. Le terme possède plusieurs dérivés, dont certains sont attestés en grec archaïque : $\tau \alpha \mu \varepsilon v v^{28}$ («être intendant»; «être questeur»), $\tau \alpha \mu 1 \varepsilon i \hat{o} v^{29}$ («trésor public»; lat. aerarium), $\tau \alpha \mu 1 \varepsilon i \alpha^{30}$

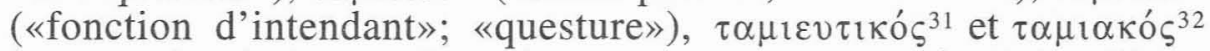

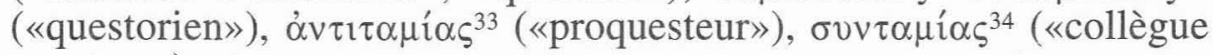
questeur»), etc.

\section{La transcription $\kappa(\mathrm{o}) v \alpha i \sigma \tau \omega \rho$}

La transcription $\kappa(0) v \alpha i \sigma \tau \omega \rho$, en revanche, est beaucoup plus rare. Elle ne se trouve, en dehors de quelques inscriptions (dont une seule est antérieure au $\mathrm{III}^{\mathrm{e}} \mathrm{S}$. ap. J.-C. $)^{35}$, chez aucun historien grec de Rome, sinon une fois dans le résumé de Dion Cassius par Zonaras (cf. infra). La plus ancienne attestation du terme se trouve chez le grammairien Hérodien, dans un développement sur la déclinaison des noms en

26 Cf. D. MAGIE, $R V$, p. 95.

27 Hdt., VIII, 51, 2; Thuc., IV, 78, 2; XéN., Hell., III, 1, 27. Cf. W. Schwahn, art. Tamiai, dans RE, IV A, 2 (1932), col. 2099-2136 (Athènes : col. 2105-2114).

$28 I G, \mathrm{I}^{3}, 590,1$ (c. 600-575 $)$; cf. Plut., Cic., 1, 4; ApP., GC, IV, 59, 257; Dion CASS., XXXVII, 52, 2.

$29 I G, \mathrm{I}^{3}, 4,14\left(485 / 4^{\mathrm{a}}\right)$; cf. POL., VI, 31, 1; DENYS, AR, VIII, 73, 5; APP., GC, I, 102, 474; DiOn CASS., LV, 32, 2.

30 Arstt., Pol., V, 7, 15, 1309 b 7; cf. Plut., Cat. Ut., 17, 1; App., GC, IV, 18, 69; DION CASS., XLI, 24, 2.

31 FL. Jos., AJ, XIV, 219 (lettre d'un magistrat romain; 44ª); DENYs, $A R$, VIII, 77, 1; SEG, 7, 1, 3 (Suse; 21 ); Plut., Cat. Ut., 16, 2, etc.

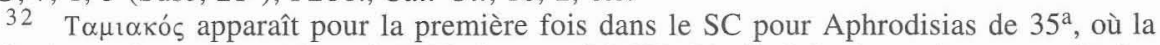

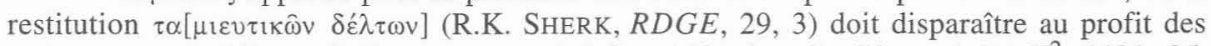

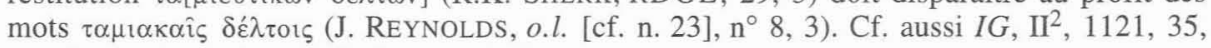
etc. Seule attestation en littérature : J. LYD., Mag., III, 70.

33 Syll. ${ }^{3}, 745,8\left(82^{\mathrm{a}}\right)$; R.K. Sherk, RDGE, 18, $90\left(81^{\mathrm{a}}\right)$; FL. Jos., AJ, XIV, 230 (lettre d'un magistrat romain; 49a); DION CASS., XLI, 43, 3.

34 Dion CASS., XLVIII, 21, 5; I. Didyma, 393, 8; 400, 8.

35 En voici le relevé, en principe complet : SEG, 6, 555, 5 (Pisid.; IIP ?); IGRR, III, 238, 4 (Galat.); IV, 1741, 16 (Lyd.); I. Eph., 677, 3; 678, 1; 710 B, 6, 25, 29; 971, 6; 3038, 8; 3084, 9; 3085, 9; Syria, 29 (1952), 317 (Arab.); Ath. Mitt., 13 (1889), p. 241 (Lyc.). Cf. aussi les dérivés к(o)vaıotípı (I. Eph., 1540, 11; ILS, 8859, 9-10 [Nysa]) et

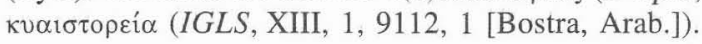


$-\tau \omega \rho^{36}$. À partir du IVe s., le mot acquiert une relative fréquence dans la littérature chrétienne, puis byzantine ${ }^{37}$. Mais il ne faut pas en conclure que l'emploi de la transcription s'est banalisé : à cette époque, le terme se rencontre dans des en-têtes de lettres adressées à des quaestores ou est

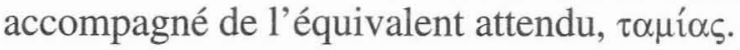

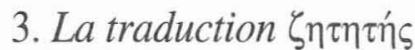

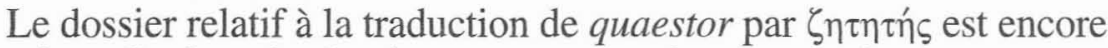
plus maigre. Seuls trois témoignages peuvent être invoqués.

\section{- Jean le Lydien}

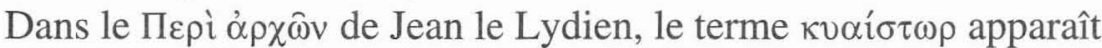
d'abord comme titre du chapitre consacré au questeur (ch. 24-29). Il débute par une citation d'un passage traduit du De officio quaestoris du

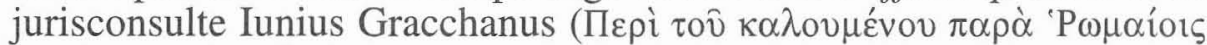

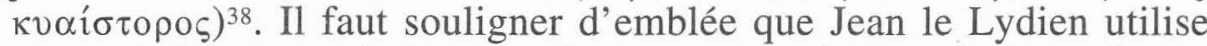
souvent des transcriptions, mais pour une raison qui donne à son témoignage une valeur particulière. Son ouvrage, dont l'érudition trahit souvent ses fonctions de professeur de latin (recours à l'étymologie, etc.) ${ }^{39}$, est avant tout un manuel d'institutions romaines. Il est donc inévitable qu'il recoure plus que tout autre à des transcriptions (ainsi,

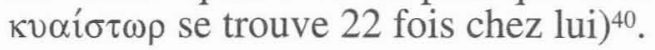

Au chapitre suivant, Jean le Lydien aborde la définition du quaestor en commençant par l'opposer, de façon inattendue, au quaesitor :

36 Partit., p. 192, 17 Boissonade. L'auteur distingue les noms d'origine grecque

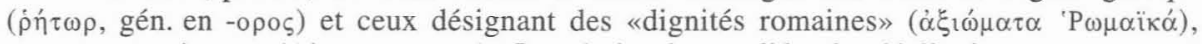
comme $\kappa v \alpha i ́ \tau \tau \omega \rho$ (gén. en - $\omega \rho \circ \varsigma$ ). Le choix du modèle de déclinaison grecque est manifestement lié au degré d'intégration du terme latin dans la langue. Ainsi, $\delta ı \kappa \tau \dot{\alpha} \tau \omega \rho$, terme banal depuis Polybe, se décline beaucoup plus souvent selon le modèle grec (gén. en

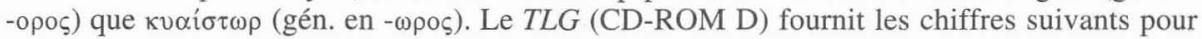
les cas obliques: $\delta 1 \kappa \tau \alpha \tau \tau_{\rho}-, 162$ occ., $\delta 1 \kappa \tau \alpha \tau \omega \rho-, 35$ occ.; $\kappa(0) \cup \alpha 1 \sigma \tau \omega \rho-(\kappa \circ 1 \alpha 1 \sigma \tau \omega \rho-$,

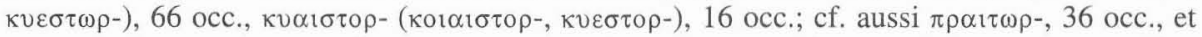

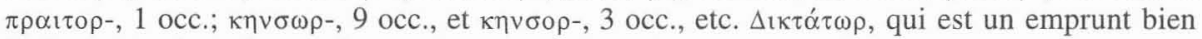
intégré, fait donc figure d'exception dans la déclinaison des noms latins en -tor transcrits en grec : cf. supra n. 13.

37 Julien, Lettres, 98, 399b; Zosime, V, 32, 6; Nilos, Lettres, II, 305; Socr., Hist. eccl., II, 34; Sozom., Id., IV, 7; Proc., Hist. secr., 14, 3, etc.

38 Cf. Dig., I, 13, 1 (Gracchanus cité par Ulpien).

39 Les méthodes de travail de l'auteur ont été étudiées récemment par M. DUBUISSON, Jean le Lydien et le latin : les limites d'une compétence, dans Serta Leodiensia secunda, Liège, 1992, p. 123-131.

40 Ceci explique aussi que nombre de transcriptions grecques ne se rencontrent pas en

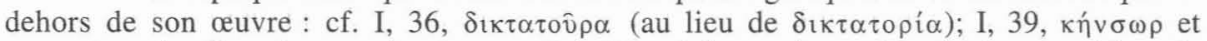

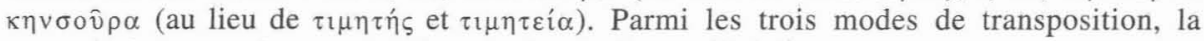
transcription y est donc surreprésentée de manière artificielle. 


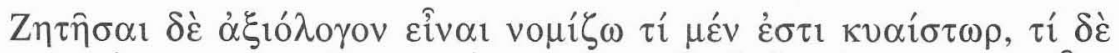

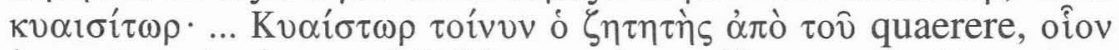

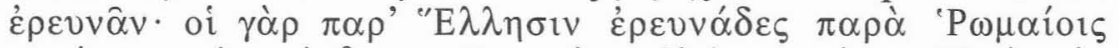

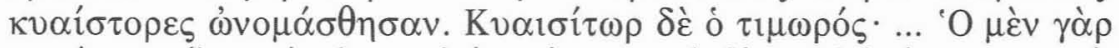

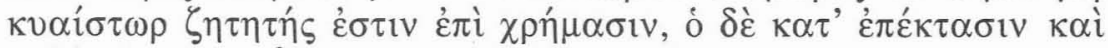

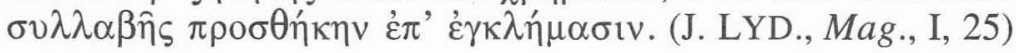

Il me paraît intéressant d'examiner ce qu'est le quaestor, ce qu'est le quaesitor... Le quaestor, c'est l'enquêteur, de quaerere «chercher»; ceux que les Grecs appelaient «enquêteurs» furent dénommés quaestores par les Romains. Le quaesitor, c'est le juge... ${ }^{41}$. Le quaestor est un enquêteur chargé des finances, l'autre, avec un allongement et une syllabe supplémentaire, un enquêteur qui s'occupe des accusations. (trad. M. Dubuisson) $)^{42}$

Pour comprendre la portée du passage, il faut bien voir que Jean le Lydien aborde la questure indirectement, par le biais de l'étymologie. Voulant établir la distinction entre quaestor et quaesitor ${ }^{43}$, il commence

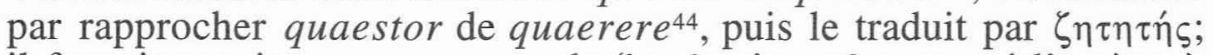

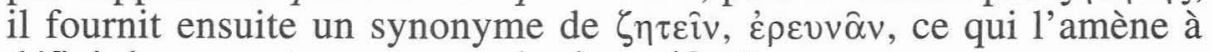

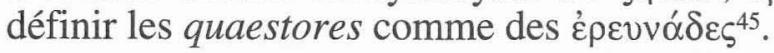

Les modernes n'ont pas relevé que l'auteur traduit aussi quaesitor

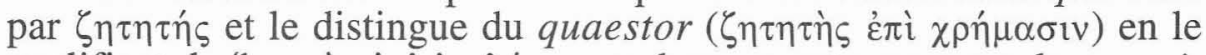

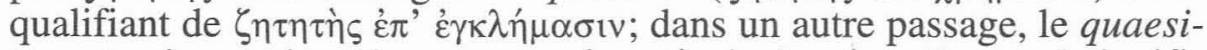

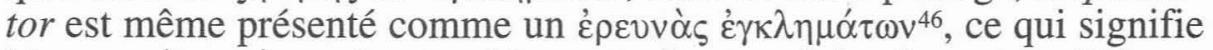

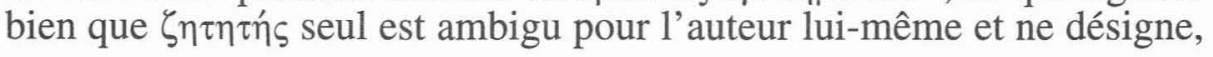

41 Jean le Lydien ajoute ici que quaestor et quaesitor ne doivent pas être confondus avec un autre mot dont la première syllabe ne s'écrit pas avec une diphtongue, mais avec une voyelle simple, et qui dérive de queror $\left(\mu \varepsilon ́ \mu \varphi \mu_{\alpha 1}\right)$ : il s'agirait de *questor («plaignant»), mais le terme n'est pas attesté en latin.

42 Je remercie M. Dubuisson de m'avoir permis de consulter l'édition de Jean le Lydien qu'il prépare en collaboration avec J. Schamp pour la Collection des Universités de France.

43 Pour quaesitor, cf. Cic., Vat., 34, etc. Aulu-Gelle, XI, 5, 1, utilise le mot pour

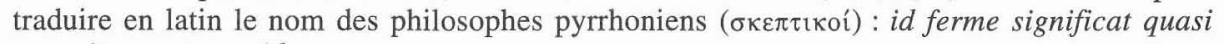
quaesitores et consideratores.

44 Pour l'étymologie de quaestor, cf. A. ERnout -A. MeIllet, Dict. étym., s.v. quaero, p. 550. Les principaux témoignages sont ceux de VARR., LL, V, 14 (quaestores a quaerendo, qui conquirerent pecunias et maleficia); PoMP., Dig., I, 2, 2, 23 (quaestores constituebantur a populo, qui rebus capitalibus praeessent); FEsTUs, s.v. quaestores, p. 310 Lindsay (Quaestores dicebantur, qui quaererent de rebus capitalibus, unde ... quaestores parricidi appellantur); autres réf. chez R. MALTBY, A Lexicon of Ancient Latin Etymologies, Leeds, 1991, s.v. quaesitor et quaestor, p. 513-514. Cf. aussi infra n. 60.

45 Il ne faut pas pour autant conclure qu'il y avait, en Grèce, des magistrats appelés

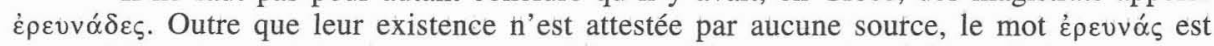
propre à Jean le Lydien, qui l'applique d'ailleurs aussi au quaesitor (cf. n. suiv.).

46 Mag., II, 29. 
comme tel, ni le quaestor, ni le quaesitor. Les deux emplois montrent

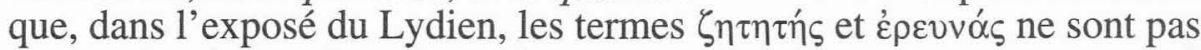
des traductions «fonctionnelles» de quaestor; ils ne servent pas à désigner le questeur en grec, mais seulement à traduire le mot latin.

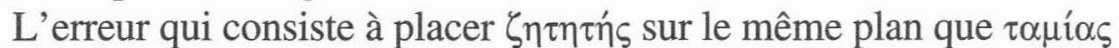
provient d'une analyse erronée du rôle de la traduction, sur laquelle D. Magie ne s'explique d'ailleurs guère. Selon lui, le grec y recourt quand il ne dispose pas d'un équivalent satisfaisant ${ }^{47}$. On ne voit plus, dès lors, le rôle que joue la transcription, censée elle aussi transposer un terme pour lequel il n'existe pas d'équivalent grec ${ }^{48}$.

L'analyse de D. Magie repose en partie sur une mauvaise appréciation de la portée de certains témoignages. Pour rendre quaestor, il existe un équivalent répandu ( $\left.\tau \alpha \mu i \alpha_{\varsigma}\right)$, que Jean le Lydien utilise d'ailleurs lui-

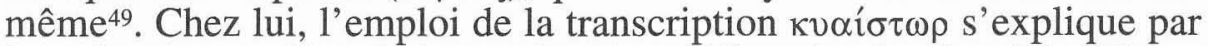
le point de vue linguistique adopté au début du chapitre 25 . Elle se rencontre encore à la fin de l'exposé sur quaestor-quaesitor, où l'auteur a soin de fournir l'équivalent ${ }^{50}$. Quant à la traduction $\zeta \eta \tau \eta \tau \eta ́ \varsigma$, , elle n'apparaît qu'une seule fois, pour faire comprendre le sens étymologique des deux substantifs dérivés de quaerere.

En définitive, le passage de Jean le Lydien montre que «questeur» ne se dit pas $\zeta \eta \tau \eta \tau \dot{n} \varsigma$ en grec. La seule information qu'il fournit est que, si l'on voulait traduire littéralement les mots quaestor et quaesitor - sans donc viser à transposer le nom d'un magistrat -, on pourrait employer $\zeta \eta \tau \eta \tau \dot{n} \varsigma^{51}$.

47 D. MAGIE, $R V$, p. 23-24.

48 ID., o.l., p. 34 .

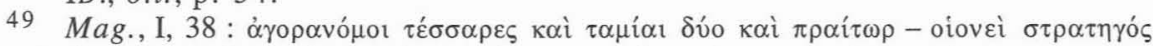

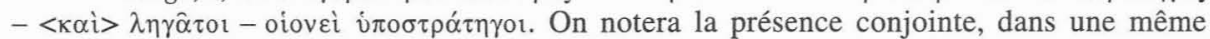
phrase, d'équivalents et de transcriptions, ces dernières étant immédiatement suivies de leur équivalent normal. Cf. aussi Mag., I, 45.

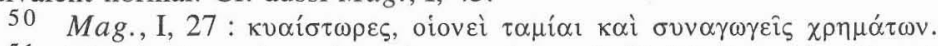

51 L'exemple $\tau \alpha \mu \alpha_{\alpha}-\zeta \eta \tau \eta \tau \varsigma_{\varsigma}-\kappa v \alpha i ́ \sigma \tau \omega \rho$ de D. Magie me paraît mal choisi à un autre titre, car $\zeta \eta \tau \eta \tau$ '́s, qui est donné par Jean le Lydien comme une traduction du mot quaestor, recouvre une réalité institutionnelle dans le monde grec classique. On n'a pas relevé qu'il existait dans l'Athènes classique des officiels appelés $\zeta \eta \tau \eta \tau \alpha$, qui constituaient une commission d'enquête non permanente intervenant dans les procès intentés contre les débiteurs du trésor public frappés d'atimie temporaire : cf. E. BERNEKER, art. $\zeta \eta \tau \eta \tau \alpha$, dans

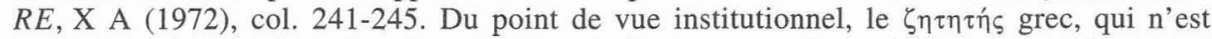
pas un magistrat, n'a guère de rapport avec le questeur, si ce n'est que tous deux sont attachés, à leur manière, à la gestion du trésor public. Si Jean le Lydien avait songé à cette institution, d'ailleurs mal connue (seuls quelques textes y font allusion), il aurait sans doute opéré le rapprochement de façon plus explicite. Un exemple mieux choisi, dont la traduction ne correspondrait à aucun titre officiel, aurait l'avantage de dissiper le doute. 


\section{- Les gloses du Corpus glossarum Latinarum}

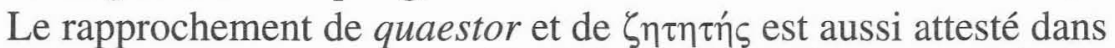

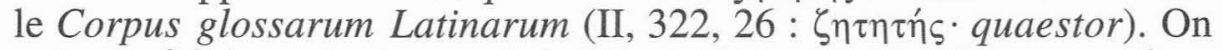
notera - fait important - que le lemme est le terme grec. Le «sens» dans lequel s'opère la transposition (grec-latin ou latin-grec) n'est pas sans incidence pour l'analyse des procédés linguistiques utilisés par les lexiques qui constituent le CGL. En effet, si les gloses font partie de lexiques bilingues, dont une des fonctions essentielles est de fournir, par définition, des traductions de mots isolés, il faut aussi apprécier la portée exacte de ces gloses, sous peine de les invoquer à mauvais escient.

Si quaestor traduit $\zeta \eta \tau \eta \tau \eta \dot{\zeta}$ dans un lexique grec-latin, dans d'autres, en revanche, le terme latin est transposé par $\tau \alpha \mu$ í $\alpha \varsigma$ (ou vice versa) ${ }^{52}$. Le recours aux deux modes de transposition (par traduction ou équivalence) s'explique par la nature des lexiques :

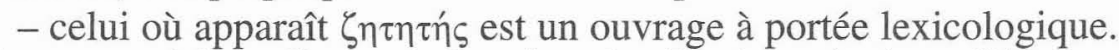

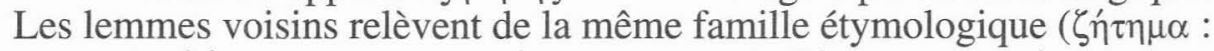

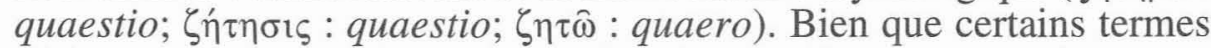
désignent la même réalité dans les deux langues ${ }^{53}$, on comprend que, par

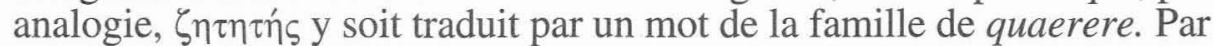
cohérence, il était obligatoire, pourrait-on dire, de donner la traduction quaestor ${ }^{54}$

- ceux qui recourent à $\tau \alpha \mu i \alpha_{\text {ç }}$ fournissent au même endroit d'autres mots dérivés du terme technique ou d'autres noms de magistrats romains ${ }^{55}$. Ce sont des lexiques spécialisés, différents du premier dans leur conception.

L'économie des lexiques du CGL ne repose donc pas sur un système unique de transposition. Le premier se borne en fait à traduire un mot grec, les autres fournissent l'équivalent grec du nom d'un magistrat romain. Cette double approche, lexicologique et institutionnelle, rend de tels témoignages incompatibles entre eux et interdit en tout cas de conclure que quaestor se disait indifféremment $\zeta \eta \tau \eta \tau \dot{n} \varsigma$ ou $\tau \alpha \mu i \alpha \varsigma^{56}$.

52 Quaestor : $\tau \alpha \mu i \alpha \varsigma$ (CGL, II, 166, 7; III, 362, 46; 415, 63); $\tau \alpha \mu i \alpha \varsigma$ : quaestor (CGL, II, 451, 23; III, 28, 18; 276, 7; 297, 62).

53 Cf., p. ex., QuinT., III, 11, 4 (à propos du terme judiciaire quaestio, que les Grecs appellent $\zeta \dot{\eta} \tau \eta \mu \alpha)$.

54 Plus généralement, il serait intéressant de voir si le mode de transposition mis en œuvre dans certains des lexiques bilingues ne dépend pas aussi, dans une certaine mesure, de leur «sens» (grec-latin ou latin-grec).

55 CGL, II, 451, 22-28 et III, 276, 5-8 : termes dérivés de $\tau \alpha \mu i \alpha_{\alpha}$; III, 28, 13-19 et III,

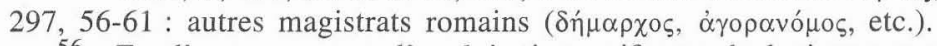

56 En d'autres termes, l'exploitation uniforme de lexiques aussi différents est une erreur analogue à celle qui consiste à considérer toutes les transpositions fournies par D. Magie comme strictement équivalentes. 


\section{- Zonaras (Dion Cassius)}

Un dernier texte, négligé par les modernes, vient confirmer

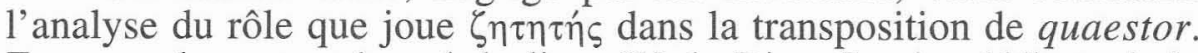
Zonaras, dans son résumé du livre IV de Dion Cassius (débuts de la République), mentionne la création de la questure :

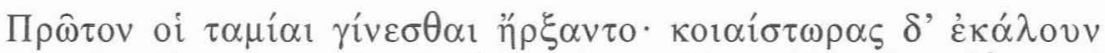

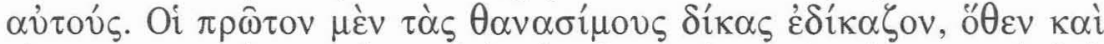

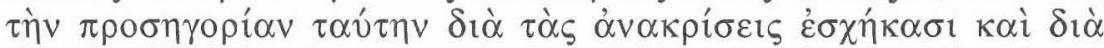

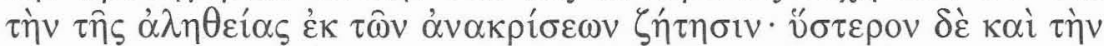

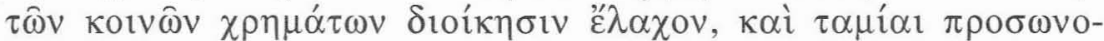
$\mu \alpha ́ \sigma \theta \eta \sigma \alpha v$. (Zon., VII, 13, 3).

Pour la première fois, on commença à désigner les $\tau \alpha \mu$ í $\alpha_{1}$, qu'on appela quaestores. À l'origine, ils instruisaient les affaires capitales; c'est de là, d'ailleurs, qu'ils ont tiré leur dénomination, qui faisait référence à leurs enquêtes, sur lesquelles se fondait la recherche de la vérité. Plus tard, ils reçurent en outre la gestion du trésor public et continuèrent à s'appeler $\tau \alpha \mu i^{\prime} \alpha 1$.

Il est impossible d'isoler avec certitude les mots de Dion Cassius

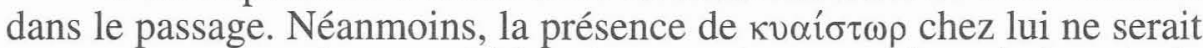
pas pour surprendre, car l'historien, en évoquant la création de la questure $^{57}$, a probablement fourni au moins une fois le nom latin du magistrat ${ }^{58}$.

Mais le principal intérêt du texte réside dans la digression de Zonaras sur le mot quaestor ${ }^{59}$. Comme Jean le Lydien, 1'auteur met le terme en relation avec la fonction primitive du magistrat. La référence à l'action de «chercher» (quaerere) procède du même souci étymologique (

57 Le texte de Zonaras doit être rapproché de Dion CASS., IV, fr. 15b Boissevain ( $\tau$ đ̀̀ $\varsigma$

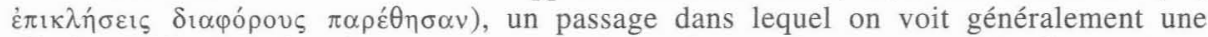
allusion à l'instauration des questeurs. L'orthographe кol í $_{\tau} \omega \rho \rho$, attestée tardivement, est peut-être due à Zonaras : cf. JULIEN, Lettres, 98, 399b; Zosime, V, 32, 6; Nilos, Lettres, II, 305; Socr., Hist. eccl., II, 34; Sozom., Id., IV, 7; Proc., Hist. secr., 14, 3, etc.

58 DION CASS., I, fr. 1,2 , souligne sa volonté de précision en matière de terminologie

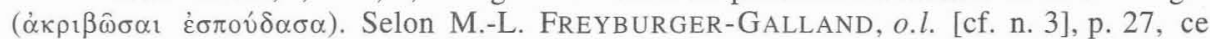
souci explique la présence de plusieurs transcriptions chez l'historien.

59 Il est difficile de dire sous quelle forme précise l'exposé se présentait chez Dion Cassius, car le raisonnement de Zonaras, tel qu'on peut le reconstituer, est passablement confus: 1. à l'origine, les nouveaux magistrats furent appelés quaestores, parce qu'ils enquêtaient sur les affaires capitales; 2 . plus tard, quand ils reçurent en plus la gestion du trésor public, on les appela aussi $\tau \alpha \mu$ í $^{\prime}$. En toute logique, on s'attendrait à trouver ici

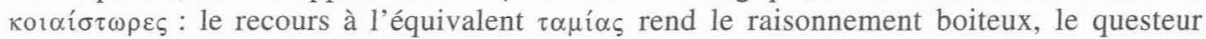
ayant toujours été désigné ainsi en grec.

60 Selon Th. Mommsen, Droit public, IV, p. 244, les témoignages de Festus et de Pomponius sont plus exacts que celui de Varron (cité supra n. 44), qui considère les 


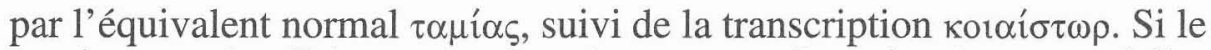

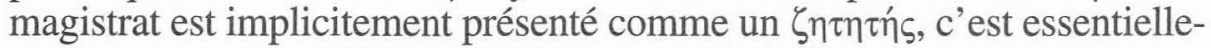
ment pour expliquer l'étymologie du terme latin ${ }^{61}$.

L'analyse des trois modes de transposition de quaestor en grec remet donc en cause le système de D. Magie et invite à formuler une conclusion différente de la sienne.

Il existe deux manières de transposer en grec les realia romains. $\mathrm{La}$ plus fréquente est, de loin, l'équivalence : quaestor se dit normalement $\tau \alpha \mu i \alpha \varsigma$. Le recours à la transcription est conditionné par des contextes particuliers. S'il n'existe pas d'équivalent, elle a toute chance de devenir la norme, même si, à l'occasion, la forme est paraphrasée dans les textes

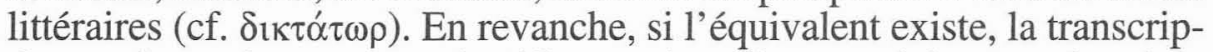
tion, qui est alors rare, se justifie par des raisons précises : volonté de fournir le terme latin (Zonaras résumant Dion Cassius) ou nécessité de l'exprimer, par exemple dans un manuel d'institutions (Jean le Lydien);

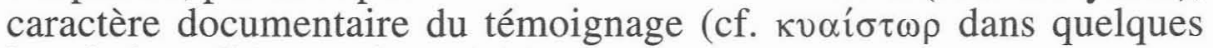
inscriptions d'époque impériale) $)^{62}$.

questeurs primitifs comme des quaestores aerarii. Même si cette fonction parait ancienne, les questeurs étaient bien chargés, à l'origine, des enquêtes criminelles (d'où le nom de quaestores parricidii); leur rôle financier résulte d'une extension de leurs attributions (assistance des consuls : cf. Th. MOMMSEN, o.l., p. 237, n. 2).

61 On se rappellera que deux gloses traduisent précisément $\zeta \dot{\eta} \tau \eta \mu \alpha$ et $\zeta \dot{\eta} \tau \eta \sigma ı \varsigma$ par quaestio (CGL, II, 322, 24-25). On retrouve aussi, pour transposer ce terme latin, la double

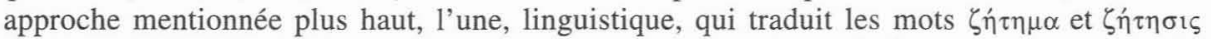
par quaestio, l'autre, fonctionnelle, qui transpose la procédure de la quaestio par $\tau 1 \mu \omega p i ́ \alpha$

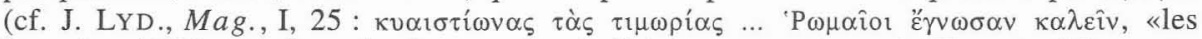
Romains décidèrent d'appeler les enquêtes judiciaires quaestiones»). Un autre fait montre

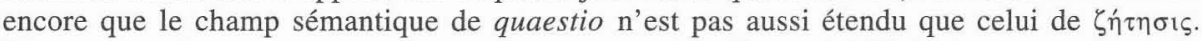
D'une part, les rares exemples de quaestio suivi d'un génitif désignent toujours la «soumission à la question»; cf. CÉs., GG, VI, 32, 2 : quaestio captiuorum; AULU-GELLE,

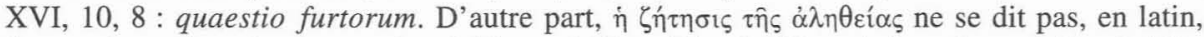
*quaestio ueri, mais, par ex., inquisitio ueri (CIC., Div., I, 13) ou indagatio ueri (ID., I, 15).

62 La transcription des mots latins en grec dans les inscriptions et les papyrus grecs mériterait une étude approfondie, qui tienne compte de tous les aspects de la question (géographique, institutionnel, chronologique, etc.). Jusqu'à présent, le phénomène a surtout été envisagé du point de vue formel (phonétique, orthographe et onomastique) : cf. W. DitTEnBERGER, Römische Namen in griechischen Inschriften und Literaturwerken, dans Hermes, 6 (1872), p. 129-155; 281-313; Th. ECKINGER, Die Orthographie lateinischer Nomina in den griechischen Inschriften, Munich, 1892; Chr. DötTLING, Die Flexionsformen lateinischer Nomina in den griechischen Papyri und Inschriften, Lausanne, 1920; B. Meinersmann, Die lateinischen Wörter und Namen in den griechischen Papyri, Heidelberg, 1927; A. CAMERON, Latin Words in the Greek Inscriptions of Asia Minor, dans AJPh, 52 (1931), p. 232-262. Pour l'Égypte, cf. S. DARIS, Il lessico latino nel greco d'Egitto, Barcelone, $1991^{2}$, en attendant la fin de la publication de l'ouvrage d'I.-M. CERVENKA-EHRENSTRASSER, Lexikon der lateinischen Lehnwörter in den griechischsprachigen dokumentarischen Texten Ägyptens mit berücksichtigung koptischer Quellen, fasc. $1(\alpha)$, Vienne, 1996. 
La traduction en grec de termes latins est un phénomène d'une tout autre nature. Elle ne vise pas, en principe, à transposer un terme (et, avec lui, la réalité ainsi désignée), mais procède d'une démarche linguistique $^{63}$. Les auteurs y recourent donc presque exclusivement pour expliquer en grec le sens d'un mot latin, après en avoir donné l'équivalent ou la transcription. L'erreur de D. Magie, commise par d'autres après lui, est de considérer qu'il s'agit d'une troisième manière effective de transposer une réalité romaine. En termes de linguistique, la traduction, peuton dire, vise presque toujours le signifiant; l'équivalent, lui, renvoie toujours au signifié 64 .

Université de Liège

Étienne FAMERIE

Sciences de l'Antiquité

Place du 20-Août, 7

$B-4000$ Liège

63 Il arrive que le grec et le latin désignent telle réalité en recourant à la même image, sans qu'il faille y voir pour autant l'influence d'une langue sur l'autre (cf., p. ex., $\chi \varepsilon i ́ \rho$ et manus au sens de «détachement [de troupes]»). Il s'agit, non pas d'une traduction, mais d'une coïncidence, les deux langues recourant indépendamment à la même métaphore («poignée d'hommes»).

64 Les conclusions auxquelles j'ai pu aboutir m'ont permis de résoudre plusieurs autres cas de «transpositions multiples» qui ont donné lieu à diverses interprétations incorrectes. L'exemple le plus frappant est celui de consul, terme pour lequel les trois transpositions

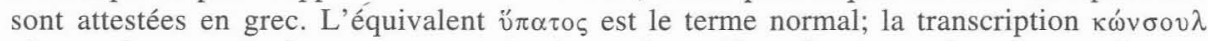
n'apparaît que pour donner le nom latin du magistrat (dans des expressions comme $0 \pi \alpha \tau$ ol, oủs 'P donnés par Denys ( $A R, \mathrm{IV}, 76,2 ; \mathrm{V}, 1,2)$ et Plutarque (Rom., 14, 3) comme des traductions possibles du mot consul, ne servent jamais à désigner le magistrat comme tel. L'opinion erronée selon laquelle $\pi$ póßovios signifierait parfois «consul» chez Appien trouve sa seule origine dans le parallélisme établi par D. Magie entre $̋ \pi \alpha \tau \circ \zeta, \kappa \omega ́ v \sigma o v \lambda$ et $\pi \rho o ́ \beta o v \lambda o \varsigma$ : cf. Ét. FAMERIE, o.l. [cf. n. 3], p. 152-159. 